\title{
Effects of Fish Oil Supplementation on Post-Resistance Exercise Muscle Soreness in Untrained Females
}

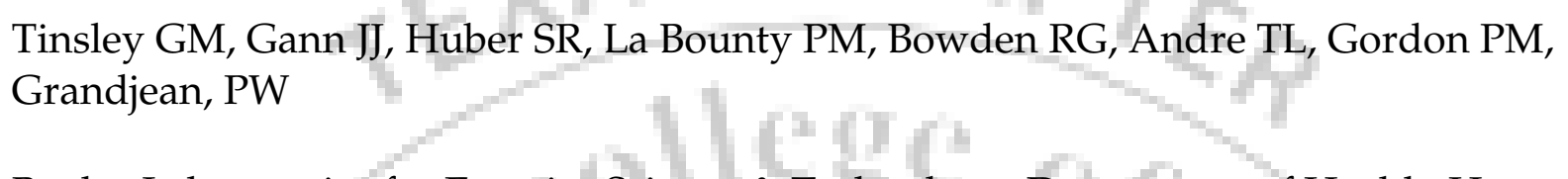

Baylor Laboratories for Exercise Science \& Technology; Department of Health, Human Performance, and Recreation; Baylor University; Waco, TX

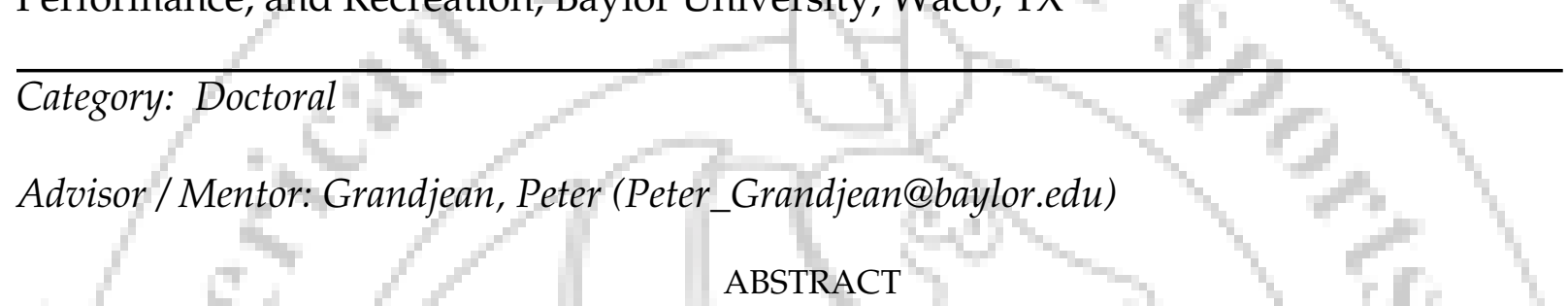

The anti-inflammatory properties of omega- 3 fatty acids contained in fish oil may help alleviate symptoms of delayed onset of muscle soreness (DOMS) and improve recovery from exercise.

PURPOSE: To examine the effects of fish oil supplementation on the time-course of post-resistance exercise muscle soreness in young untrained females.

METHODS: Seventeen non-resistance trained females (age $23.6 \pm 3.0$ y, \% fat $27.4 \pm 3.2$, weight $60.2 \pm 9.6$ $\mathrm{kg}$ ) were randomized into one of two groups: fish oil ( $6 \mathrm{~g} / \mathrm{d}$; 5:1 EPA:DHA) or placebo $(6 \mathrm{~g} / \mathrm{d}$ corn/soy oil). After consuming the supplements for one week, participants underwent a single bout of resistance exercise designed to induce muscle damage. Subjects performed 10 sets to failure of biceps curl machine and leg extensions using 50\% of the previously measured 1-repetition maximum. Over the next week, subjective muscle soreness of the upper and lower body was measured via a grounded 10-cm visual analog scale. At 48 hours and 1 week post-exercise, subjects performed a test to determine soreness during functional movements. The comparison-wise error rate was set at $\mathrm{p}<0.10$.

RESULTS: Muscle soreness increased significantly in both groups and peaked at 48 hours post-exercise. The fish oil group perceived less muscle soreness in the lower body than the placebo group $(p=0.06)$, but there was no difference in the upper body $(p=0.27)$. The fish oil group reported less perceived soreness during functional movements $(\mathrm{p}=0.07$ for upper and lower body soreness). Effect sizes, indicating the reduction in muscle soreness that may be attributed to fish oil ( $\Delta$ effect size between groups), was 0.75 $(95 \%$ CI: $-0.70-2.20)$ for the arms and $0.77(-0.76-2.33)$ for the legs. The effect size for the functional tests was $0.63(-0.71-1.97)$ for the arms and $0.57(-0.85-1.99)$ for the legs.

CONCLUSION: Supplementing the diet with 6 grams per day of fish oil may alleviate the muscle soreness experienced after resistance training in young untrained females, but additional studies with larger sample sizes should be conducted to confirm these findings.

This research was supported by Baylor University, and supplements were donated by The Cooper Institute, Dallas, TX.

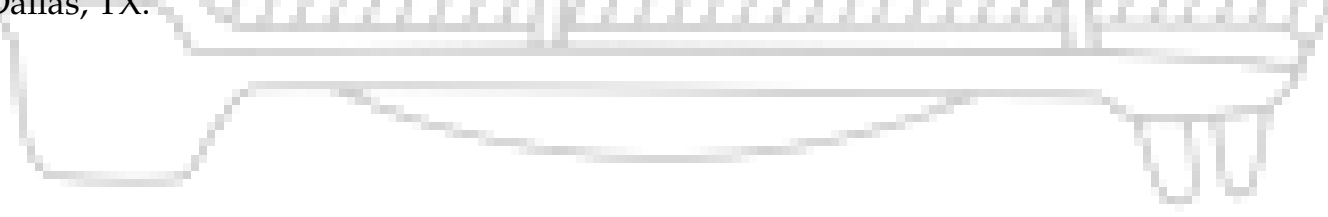

